\title{
Moving towards a cure in genetics: what is needed to bring somatic gene therapy to the clinic?
}

\author{
Martina C. Cornel $\mathbb{C}^{1} \cdot$ Heidi C. Howard ${ }^{2} \cdot$ Daniel Lim $^{3} \cdot$ Vence L. Bonham $^{4} \cdot$ Kirmo Wartiovaara $^{5}$
}

Received: 7 October 2018 / Accepted: 7 November 2018 / Published online: 19 December 2018

(c) European Society of Human Genetics 2018

\begin{abstract}
Clinical trials using somatic gene editing (e.g., CRISPR-Cas9) have started in Europe and the United States and may provide safe and effective treatment and cure, not only for cancers but also for some monogenic conditions. In a workshop at the 2018 European Human Genetics Conference, the challenges of bringing somatic gene editing therapies to the clinic were discussed. The regulatory process needs to be considered early in the clinical development pathway to produce the data necessary to support the approval by the European Medicines Agency. The roles and responsibilities for geneticists may include counselling to explain the treatment possibilities and safety interpretation.
\end{abstract}

\section{Introduction}

Monogenic conditions have traditionally been considered incurable. Somatic gene therapy (SGT) and especially gene editing (e.g., with clustered regularly interspaced short palindromic repeats-CRISPR-associated 9 (CRISPRCas9)) have generated new hope for improved treatment. Indeed, SGT approaches are currently being tested and some are offered to patients (Table 1). Especially for gene editing, concerns still remain, for example, regarding immunological and general safety as well as questions of equity and economics.

During a workshop at the European Human Genetics Conference 2018, we discussed how SGT may change the

Martina C. Cornel

mc.cornel@vumc.nl

1 Amsterdam UMC, Clinical Genetics and Amsterdam Public Health Research Institute, Section Community Genetics, Vrije Universiteit Amsterdam, De Boelelaan 1117, Amsterdam, The Netherlands

2 Centre for Research Ethics and Bioethics, Uppsala University, Box 564, Uppsala 751 22, Sweden

3 Kirkland \& Ellis International LLP, London, UK

4 Social and Behavioral Research Branch National Human Genome Research Institute, National Institutes of Health, Bethesda, MD, USA

5 Helsinki University Hospital, Clinical Genetics, University of Helsinki, Meilahdentie 2, Helsinki 00290, Finland face of medicine, especially for rare genetic diseases. We discussed:

- The current state of science, especially for genetic editing;

- The legal and regulatory challenges;

- Equity in access; and

- The potential role of geneticists.

\section{Trials using somatic gene editing: the current status}

The treatment of genetic diseases using gene editing is tempting in its simplicity: instead of treating the symptoms, we could correct the cause. In comparison with traditional gene therapy, the edited gene would be under normal genetic regulators. SGT may also circumvent the ethical, legal and safety problems of germline modifications. As of August 2018, there are a handful of approved SGT products on the market, none of which use gene editing.

The results from current clinical trials with zinc-finger (ZFN) and transcription activator-like effector (TALE) nucleases are promising but still only preliminary. For example, CCR5 (and other) HIV receptor genes have been removed from patient blood cells in vitro, resulting in lower Human Immunodeficiency (HI) virus titre when the cells are re-introduced in the patient [1]. In November 2017, the first in vivo gene editing clinical trial started for a metabolic disease, mucopolysaccharidosis II, with ZFNs administered 


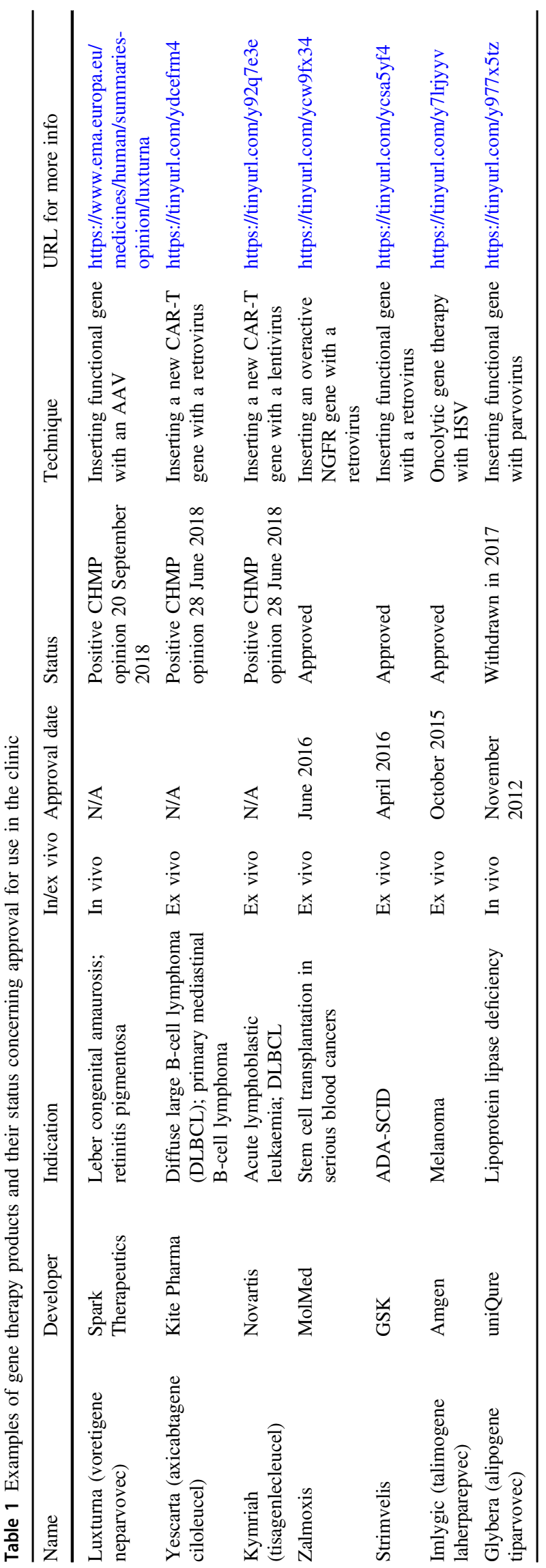

directly in the patient [2]. The first CRISPR-Cas9 clinical trials are underway in China for sporadic cancers and are planned or started in the United States and the European Union for haemoglobinopathies [2].

Importantly, it is likely that the fastest advancements of SGT and information on safety will first come from the cancer field, where the editing is often targeted to immune cells for better therapeutic effect. This may not directly tell us about the efficacy of treating specific inherited diseases, but will give valuable data about the feasibility, treatment potential and risks of using SGT in patients. The first attempts and successes in genetic editing of cells in inherited diseases will most likely continue to be developed in the haematological and metabolic fields.

\section{What is needed to launch genome editing in the clinic?}

The development and sale of human medicinal products is, rightly, one of the most highly regulated industries in the world. As a general rule, medicinal products are required to go through rigorous testing to establish the safety and efficacy of the product to the satisfaction of the regulatory bodies charged with overseeing the industry. Gene therapy medicinal products (GTMPs) are no exception to this requirement, and present particular challenges for developers and regulators alike. They must balance the twin imperatives of ensuring the safety, efficacy and durability of such treatments, while also providing an efficient regulatory service that facilitates access to potentially life-changing and curative medicines for those in dire need of new treatments.

In Europe, GTMPs must be approved centrally by the European Medicines Agency (EMA) and fall within the broader classification of "advanced therapy medicinal products" (ATMPs). Along with GTMPs, there are two further subclasses of ATMP: somatic cell therapy medicinal products (sCTMPs) and tissue engineered products (TEPs). The EMA has created a specific committee-the Committee for Advanced Therapies (CAT) — to specialise in assessing ATMPs and advising the Committee for Medicinal Products for Human Use (CHMP) (and through them, ultimately advise the European Commission) as to whether marketing approval should be granted for such products [3].

The evidence that a developer of a therapy is required to submit for consideration of a marketing approval takes the form of a detailed data package comprising preclinical, clinical, pharmacokinetic/pharmacodynamic and other data. The clinical development pathway (traditionally divided into preclinical, phase I, phase II and phase III studies) is directed towards producing the data necessary to produce 
the technical package to support the approval of the product.

However, as Scott Gottleib, FDA Commissioner, noted in a keynote address to the Alliance for Regenerative Medicine on 22 May 2018 [4], unlike traditional drug review of small molecule and biologic therapies,

"where $80 \%$ of the review is focused on the clinical portion of that process, and maybe $20 \%$ is focused on the product issues...this general principal is almost completely inverted when it comes to cell and gene therapy".

The establishment of clear clinical efficacy at an early stage even in small groups of patients, offset by the vastly increased complexity of the product manufacturing and quality control considerations, represents a real challenge to the paradigms of the existing review pathway (in which statistically significant clinical efficacy can be marginal, but the product and quality issues are fairly straightforward). Regulators have recognised this, and the Food and Drug Administration (FDA) in the United States released a set of six new draft guidelines on gene therapy on 11 July 2018 [5], shortly followed 2 days later by the EMA releasing a substantial revision to its existing 2001 guidance on GTMPs on 13 July 2018 [6]. It remains to be seen, however, how these guidelines are translated into regulatory practice as the new wave of gene therapy treatments progress through the development and approval pathway.

\section{Willingness to participate in clinical trials for sickle cell disease}

Approximately 5\% of the world's population carries variants for haemoglobin disorders, mainly, sickle cell disease (SCD) and thalassaemia [7]. Globally, 300,000-400,000 babies with SCD are born each year, adding up to $14,242,000$ affected births between 2010 and 2050 [8]. Migration has increased the number of individuals living with SCD in Northern Europe and the United States. While SCD was identified over a 100 years ago, limited treatments are available worldwide. A need for improved health care is recognised for this chronic disease with severe health complications and life expectancy reduced on average by 30 years [9].

SCD is caused by a single point mutation in the sixth codon of the beta-haemoglobin subunit. The molecular mechanism of SCD has been identified as a potential target for somatic gene editing. Two approaches to reduce the burden and/or cure SCD using gene editing are currently being investigated: correcting the beta-globin point mutation [10] and targeting the BCL11A gene for foetal haemoglobin reinduction [11], leading to higher levels of foetal haemoglobin and reduced disease severity. In January 2018,
National Institutes of Health (NIH) in the United States launched a Somatic Cell Genome Editing program to develop quality tools to perform effective and safe somatic genome editing in human patients. These research tools will be made widely available to the research community to reduce the time and cost required to develop new therapies [12].

On 31 August 2018, the first CRISPR clinical trial for $\beta$ thalassaemia backed by two US companies was launched in Regensburg, Germany [13]. On 13 September 2018, the US National Heart, Lung, and Blood Institute at the NIH announced the Cure Sickle Cell Initiative to support research in SGT [14]. During the ESHG workshop, Vence Bonham (see author list) reported on community-engaged research to inform regulators, biotech companies and researchers of the perspectives of the US sickle cell disease community regarding their willingness to participate in clinical trials and their views of the potential that gene editing can be a curative treatment. Bonham reported that a major concern is whether the SCD patient population will have equitable access to SGT as a treatment option in clinical care. The US NIH is taking an important step to make the promise of a cure for this debilitating disease a reality.

\section{Will geneticists move to treatment?}

In the translation process of new technologies from the research to the clinical context, the question of which stakeholder group (s) will take on which roles and responsibilities must be addressed in order to ensure a responsible offer of services. In practice, relevant and pressing questions for somatic gene editing include: Who will be the healthcare professionals involved in the offer of SGT? In particular, given the (historical) central role clinical geneticists have played in diagnosing genetic disease, we are particularly interested in the question: what role, if any, will or should clinical geneticists play in the offer of such treatments? If the assessment of safety of SGT includes whole genome sequencing and analysis, will clinical geneticists be involved in the interpretation and communication? As of now, very little guidance can be found in the academic literature or in policy documents to answer these questions. Roles and responsibilities of clinical geneticists and genetic counsellors do not exclude treatment activities. They could include counselling for SGT or helping to establish adequate ethical frameworks. Furthermore, genetics laboratories may also assume responsibility to search for off-target events for SGT services.

\section{Conclusion}

Clinical trials for somatic cell gene editing are currently underway. While preliminary results are promising, 
questions about safety, equity and responsible use in vulnerable populations remain. Furthermore, the regulatory pathway leading to the development and approval of such novel GTMPs appears difficult to navigate. For clinical geneticists there is an apparent opportunity.

\section{Compliance with ethical standards}

Conflict of interest The authors declare that they have no conflict of interest.

\section{References}

1. Tebas P, Stein D, Tang WW, Frank I, Wang SQ, Lee G, et al. Gene editing of CCR5 in autologous CD4 T cells of persons infected with HIV. N Engl J Med. 2014;370:901-10.

2. Dunbar CE, High KA, Joung JK, et al. Gene therapy comes of age. Science. 2018;359:eaan4672.

3. Committee for Advanced Therapies (CAT). http://www.ema. europa.eu/ema/index.jsp?curl=pages/about_us/general/general_ content_000266.jsp\&mid=WC0b01ac05800292a4. 2018 Accessed 2 Sept 2018.

4. Gottlieb S. Remarks by Commissioner Gottlieb to the Alliance for Regenerative Medicine's Annual Board Meeting. https://www. fda.gov/NewsEvents/Speeches/ucm608445.htm. 2018 Accessed 2 September 2018.

5. U.S. Food and Drug Administration. Cellular \& Gene Therapy Guidances. https://www.fda.gov/biologicsbloodvaccines/ guidancecomplianceregulatoryinformation/guidances/cellulara ndgenetherapy/default.htm. 2018 Accessed 2 September 2018.

6. European Medicines Agency. Guideline on the quality, nonclinical and clinical aspects of gene therapy medicinal products. http://www.ema.europa.eu/docs/en_GB/document_library/ Scientific_guideline/2018/07/WC500252056.pdf. 2018 Accessed 2 September 2018.

7. World Health Organization. Regional Office for Africa. Sickle cell disease. Fact sheet. https://www.afro.who.int/health-topics/sicklecell-disease. 2017 Accessed 12 August 2018.

8. Piel FB, Hay SI, Gupta S, Weatherall DJ, Williams TN. Global burden of sickle cell anaemia in children under five, 2010-2050: modelling based on demographics, excess mortality, and interventions. PLoS Med. 2013;10:e1001484.

9. Piel FB, Steinberg MH, Rees DC, et al. Sickle cell disease. N Engl J Med. 2017;376:1561-73.

10. Vakulskas CA, Dever DP, Rettig GR, et al. A high-fidelity Cas9 mutant delivered as a ribonucleoprotein complex enables efficient gene editing in human hematopoietic stem and progenitor cells. Nat Med. 2018;24:1216-24.

11. Antoniani C, Meneghini V, Lattanzi A, et al. Induction of fetal hemoglobin synthesis by CRISPR/Cas9-mediated editing of the human $\beta$-globin locus. Blood. 2018;131:1960-73.

12. National Institutes of Health. Somatic Cell Genome Editing. https:// commonfund.nih.gov/editing. 2018 Accessed 12 August 2018.

13. Gottlieb S. A safety and efficacy study evaluating CTX001 in subjects with transfusion-dependent $\beta$-thalassemia. https://clinicaltria 1s.gov/ct2/show/NCT03655678. 2018 Accessed 29 September 2018.

14. NIH launches initiative to accelerate genetic therapies to cure sickle cell disease. https://www.nih.gov/news-events/news-relea ses/nih-launches-initiative-accelerate-genetic-therapies-curesickle-cell-disease. 2018 Accessed 29 September 2018. 